\title{
Elevated Glucagon in a Patient with Necrolytic Acral Erythema: A Case Report and Review of the Literature
}

This paper provides an interesting and thorough description of a specific case of hepatitis $C$ infection with necrolytic acral erythema, contributing novel information to the limited existing knowledge about this condition. With high originality and significance, the investigation gives a well-structured account of the improvement seen in the patient

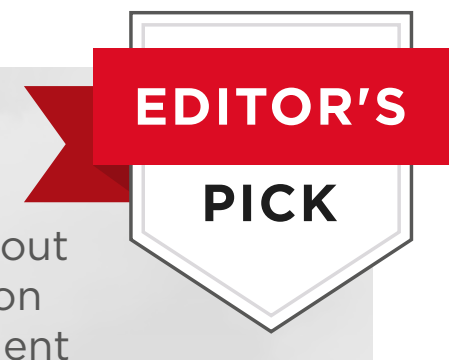
following oral zinc replacement therapy, alongside a review of currently available necrolytic acral erythema case literature.

Authors:

Disclosure:

Received:

Accepted:

Keywords:

Citation:
*Jenna E Koblinski, ${ }^{1}$ Blake W Traube, ${ }^{1}$ Margaret Kessler, ${ }^{2}$ Brenda Shinar ${ }^{1,2}$

1. The University of Arizona College of Medicine - Phoenix, Arizona, USA

2. Banner University Medical Center - Phoenix, Arizona, USA

*Correspondence to jennakoblinski@email.arizona.edu

The authors have declared no conflicts of interest.

05.02.21

30.03 .21

Dermatology, gastroenterology, glucagon, hepatitis, hepatology, necrolytic acral erythema (NAE).

EMJ Hepatol. 2021;9[1]:47-56.

\section{Abstract}

Necrolytic acral erythema (NAE) is a relatively newly described dermatologic disease that is often associated with hepatitis $\mathrm{C}$ virus (HCV). Oral zinc therapy is a successful treatment; however, therapy is often delayed due to misdiagnosis. There are limited reports of NAE in the literature. This paper presents a case of NAE in a 68-year-old male with untreated HCV, whose NAE was diagnosed and treated as recurrent cellulitis for 12 years. He had low serum zinc and elevated serum glucagon levels. Elevated glucagon is not often reported in NAE, but the patient's CT abdomen was negative, ruling out glucagonoma and necrolytic migratory erythema. He improved with oral zinc replacement and was referred to the hepatology department for HCV treatment. This paper additionally presents a review of the literature for NAE cases.

\section{INTRODUCTION}

Necrolytic acral erythema (NAE) was first described in 1996 by el Darouti and Abu el Ela in Egypt. ${ }^{1}$ It is most often associated with hepatitis $\mathrm{C}$ virus (HCV) infection and is similar in morphology to other necrolytic erythemas. ${ }^{2}$ Classically, NAE presents as well-demarcated, dusky erythematous plaques over the dorsum of the feet and toes, with thickening of the 
plaques over time; 3 however, NAE rarely develops in other areas such as the hands and elbows as well. ${ }^{2}$ While there have been more reports of NAE since its original designation, there is still a paucity of data regarding this entity. A case of NAE associated with elevated glucagon levels in a 68-year-old male with untreated $\mathrm{HCV}$ is presented here. His NAE was diagnosed and treated as recurrent cellulitis for 12 years.

\section{CASE REPORT}

The patient was a 68-year-old white, nonHispanic male with a past medical history of untreated HCV, ex-intravenous (IV) drug abuse, chronic venous insufficiency, hypertension, tinea pedis, and recurrent left foot cellulitis, who was admitted to the hospital for progressive worsening of a painful left foot plaque. He reported that the lesion had started 3-4 weeks prior, but that he had experienced intermittent episodes of the same rash for the past 12 years, which had resolved with oral antibiotics, topical ketoconazole, and topical clotrimazole-betamethasone. The current plaque began localised to the left third digit and was associated with a burning pain. Prior to his current admission, he had been seen by multiple providers and had been unsuccessfully treated with oral cephalexin and topical mupirocin, doxycycline, and topical clotrimazole, and finally, IV vancomycin. Due to progression of his disease to his remaining left digits and proximal left foot, and new-onset redness and pain in his right foot, he was evaluated by podiatry who recommended he come to the emergency department for evaluation. He denied recent trauma or burns to his feet. His review of systems was not significant.

His past medical history was significant for untreated-HCV, ex-IV drug abuse, chronic venous insufficiency, hypertension, history of tinea pedis, and recurrent left foot cellulitis. He was diagnosed with HCV approximately 40 years prior and had never received treatment due to transportation and insurance barriers. His only medication was lisinopril.

On initial physical examination (Figure 1 and Figure 2 ) by the internal medicine team he had a well-demarcated, erythematous, ulcerated plaque extending to the dorsal surface with increased oedema on his left foot. There were macerations, fissuring, and sloughing of the epidermis at the plantar surfaces near the digits on both feet. Thickened nail plates with yellow discolouration and subungual debris were seen on multiple toenails, bilaterally. His leukocyte count and creatinine were elevated, and his serum albumin was low. His HCV antibody screen was $>11$ index (normal: $\leq 0.79$ ) and his HCV RNA was 13,800,000 international units/mL (normal: <15 international units $/ \mathrm{mL}$ ). The patient was started on IV vancomycin and piperacillin/tazobactam, as well as oral terbinafine and topical clotrimazolebetamethasone for presumed left foot infection and right hallux infection. An X-ray was ordered, demonstrating some concern for osteomyelitis; a follow-up MRI was negative.

Due to failure to respond to antimicrobial therapy, dermatology was consulted on hospital Day 5. His physical examination at that time demonstrated dark erythema with superficial desquamation of bilateral dorsal and plantar feet and toes. Multiple toenails were thickened, with yellow discolouration and subungual debris. In addition, his bilateral hands, elbows, and heels had keratotic erythematous plaques with additional fissures and desquamation of his hands. He had fingernail clubbing. The remainder of his skin was clear. In addition to NAE, the differential diagnosis included lichen planus, psoriasis, atopic dermatitis, necrolytic migratory erythema (NME), niacin deficiency, acrodermatitis enteropathica, eczematous dermatoses, and tinea pedis.

The diagnosis of NAE was suspected based on clinical presentation of the hyperkeratotic plaques affecting his acral surfaces, as well as with his history of $\mathrm{HCV}^{2}$ This diagnosis also remained the most likely when working through the differential diagnoses. While lichen planus does have a similar distribution, it has a characteristic violaceous colour and polygonal shape not seen in the patient. ${ }^{4}$ Psoriasis was not strongly considered as the patient's rash did not have the typical silvery scale of psoriasis, but rather a very superficial desquamation. ${ }^{4}$ NME affects the perioral and peri-genital regions as well as the extremities (discussed below). ${ }^{5}$ 


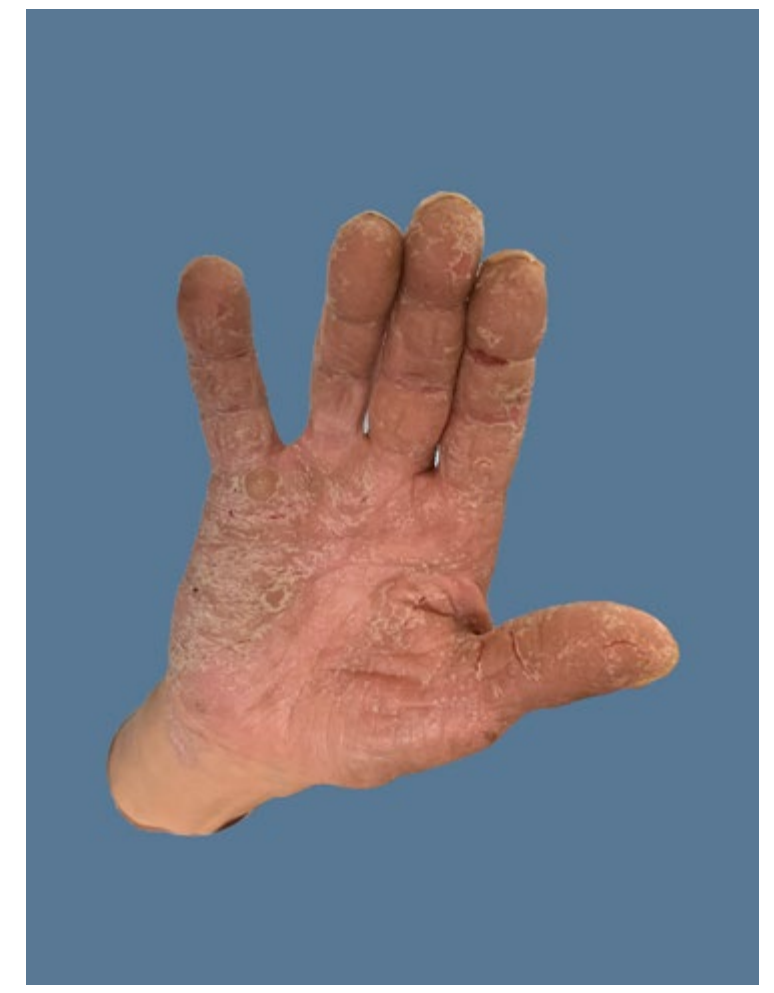

Figure 1: Necrolytic acral erythema affecting the right hand.

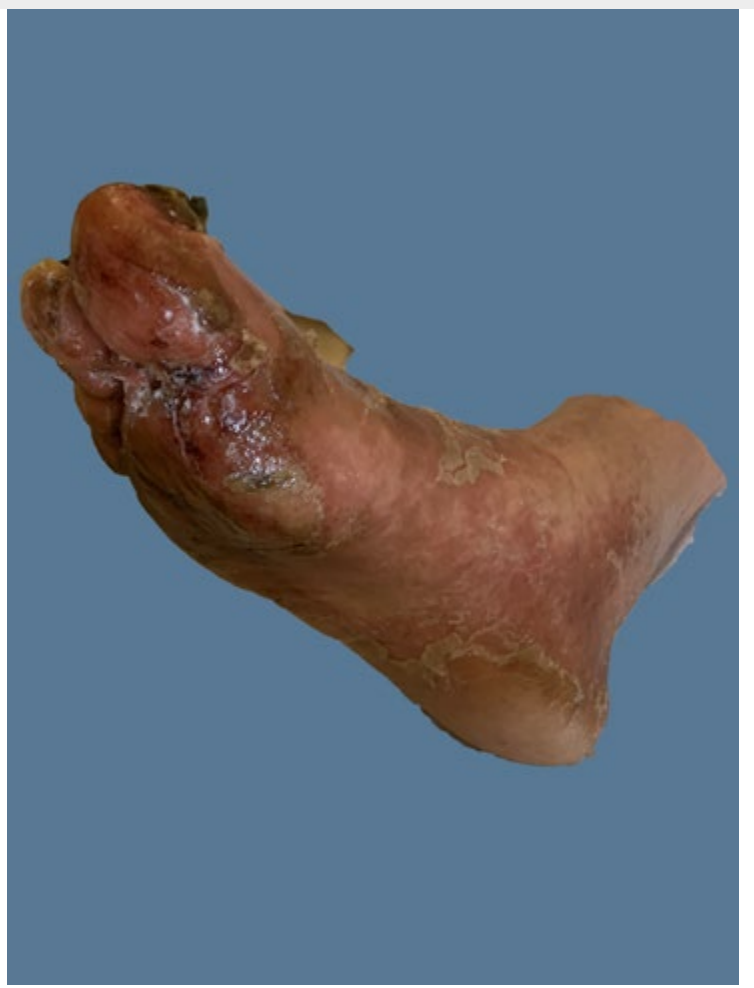

Figure 2: Necrolytic acral erythema affecting the right foot. 
Acrodermatitis enteropathica has a periorificial and peri-acral distribution and also often presents as a triad with diarrhoea and alopecia. ${ }^{4}$ Niacin deficiency can result in pellagra, which is characterised by the triad of dermatitis (typically affecting the neck or chest and hands), diarrhoea, and dementia. ${ }^{4}$ Simple eczema should also be included, but the rash was not blistering or oedematous. ${ }^{4}$ Tinea pedis was also considered; however, tinea does tend to be more inflammatory, with intense pruritus, brighter erythema, and finer scale (rather than the broad sheets of scale the patient had on his feet). ${ }^{4} \mathrm{He}$ also had repeatedly failed treatment for tinea. While NAE was suspected, a biopsy was performed for more definitive diagnosis and laboratory tests were ordered for further evaluation.

The pathology report showed psoriasiform dermatitis with slight pallor in the superficial epidermis with foci of parakeratosis and serum, which was compatible with NAE given the corresponding clinical features. His glucagon level was elevated at $96 \mathrm{pg} / \mathrm{mL}$ (normal: 8-57 $\mathrm{pg} / \mathrm{mL}$ ) and his zinc level was low at $41 \mathrm{mcg} /$ dL (normal: $60-130 \mathrm{mcg} / \mathrm{dL}$ ). His cryoglobulin screen was negative and his rheumatoid factor, C3, and C4 levels were within normal limits. The clotrimazole-betamethasone, antibiotics, and terbinafine were discontinued and he was started on a zinc supplement and topical clobetasol. The patient improved to the point of being able to ambulate and there was no longer visible skin sloughing. He was discharged and scheduled for hepatology follow-up and a CT abdomen to definitively rule out glucagonoma due to his elevated glucagon level. The CT abdomen was negative for pancreatic lesions, which again verified the diagnosis of NAE as opposed to NME; the CT was also negative for hepatic cirrhosis and hepatocellular carcinoma. The patient is currently being seen by the hepatology service, with plans to start treatment for his $\mathrm{HCV}$.

\section{DISCUSSION}

NAE is a relatively newly described pathology and is often associated with HCV. ${ }^{1}$ While it was once believed to be pathognomonic for $\mathrm{HCV}$ infection, it has since been reported in seronegative patients as well. ${ }^{3,6}$ While the exact incidence and prevalence of NAE is unknown, the majority of cases have been reported from Egypt, which has the highest prevalence of $\mathrm{HCV}$ in the world. ${ }^{7}$ Since its initial description in Egyptian patients, it has been reported in other countries, including the USA, ${ }^{3}$ India, ${ }^{6}$ Pakistan, ${ }^{8}$ and Canada, among others. ${ }^{9}$

On $13^{\text {th }}$ January 2021 , a literature search of the PubMed database was conducted using the search term 'Necrolytic Acral Erythema'; all cases written in English were included (Table 1).,3,6,947 There were 42 reports found, with a total of 63 cases of NAE described. Of these cases, 16 patients were black, two were white, three were Asian, and 42 did not explicitly list the patient's race or ethnicity. The presented patient is white, non-Hispanic, which contributes to the epidemiology of NAE as it has mainly been described in the black population, although there is a paucity of data on race in the reports. Fifteen of the 16 cases reported in the black population were reports from the USA; this is likely because in the USA, HCV is more prevalent in African Americans than any other racial group. ${ }^{48}$ For the patients with an HCV diagnosis, it is unclear how long they had had the infection. At 68 years old, the patient is the same age as one patient and older than all but two of the reported cases; he had untreated HCV for approximately 40 years. While the exact pathogenesis of NAE is not yet known, it is hypothesised to be multifactorial, with contributions from zinc deficiency, hypo/ hyperglucagonaemia, hepatic dysfunction, and hypoalbuminaemia, among others. ${ }^{2}$ The patient's extensive history of untreated HCV, paired with his recurrent NAE, may suggest an association between infection duration and NAE. More studies need to be completed to evaluate for risk factors in NAE aetiopathogenesis.

As aforementioned, NAE can be associated with an array of laboratory abnormalities. The patient had hypoalbuminaemia, zinc deficiency, and hyperglucagonaemia. Interestingly, NAE has been demonstrated to be responsive to zinc therapy in patients with both normal zinc levels ${ }^{10,11}$ and zinc deficiency. ${ }^{13,28}$ The patient was found to be zinc deficient and improved with zinc supplementation, contributing to the proposed link between zinc deficiency and NAE. Zinc levels should be checked in all patients with suspected NAE. 
Table 1: PubMed-indexed cases of necrolytic acral erythema.

\begin{tabular}{|c|c|c|c|c|c|c|c|c|}
\hline Study & Patient & $\begin{array}{l}\text { Age } \\
\text { (years); } \\
\text { sex }\end{array}$ & Ethnicity & $\begin{array}{l}\mathrm{HCV} \\
\text { status }\end{array}$ & $\begin{array}{l}\text { Serum } \\
\text { zinc } \\
\text { level }\end{array}$ & \begin{tabular}{|l} 
Serum \\
glucagon \\
level
\end{tabular} & Treatment & $\begin{array}{l}\text { Other diseases in } \\
\text { patient }\end{array}$ \\
\hline \multirow[t]{7}{*}{$\begin{array}{l}\text { el Darouti et al., } \\
1996\end{array}$} & 1 & 55; F & $\mathrm{N} / \mathrm{A}$ & Positive & Normal & $N / A$ & $\begin{array}{l}\text { Zinc and amino } \\
\text { acids }\end{array}$ & $N / A$ \\
\hline & 2 & $12 ; \mathrm{F}$ & $N / A$ & Positive & Normal & $\mathrm{N} / \mathrm{A}$ & $\begin{array}{l}\text { Zinc and amino } \\
\text { acids }\end{array}$ & $N / A$ \\
\hline & 3 & $45 ; M$ & $\mathrm{~N} / \mathrm{A}$ & Positive & Normal & $\mathrm{N} / \mathrm{A}$ & $\begin{array}{l}\text { Zinc and amino } \\
\text { acids }\end{array}$ & $N / A$ \\
\hline & 4 & $38 ; M$ & $N / A$ & Positive & $N / A$ & $N / A$ & IFN- $a$ and zinc & $N / A$ \\
\hline & 5 & $50 ; M$ & $N / A$ & Positive & $N / A$ & $N / A$ & $\begin{array}{l}\text { Liver } \\
\text { supplements }\end{array}$ & $N / A$ \\
\hline & 6 & 40; F & $N / A$ & Positive & Normal & $N / A$ & $\begin{array}{l}\text { Zinc and amino } \\
\text { acids }\end{array}$ & $N / A$ \\
\hline & 7 & 35; F & N/A & Positive & $N / A$ & $N / A$ & IFN-a & DM \\
\hline $\begin{array}{l}\text { Khanna et al., }{ }^{10} \\
2000\end{array}$ & 1 & 43; F & Black & Positive & Normal & Normal & IFN and zinc & $\begin{array}{l}\text { Atopic dermatitis, } \\
\text { asthma, allergic } \\
\text { rhinitis }\end{array}$ \\
\hline $\begin{array}{l}\text { Hivnor et al.,"1 } \\
2004\end{array}$ & 1 & 11; F & Black & Positive & Normal & Normal & IFN and ribavirin & $\begin{array}{l}\text { HCV-associated } \\
\text { nephrotic syndrome }\end{array}$ \\
\hline \multirow[t]{5}{*}{$\begin{array}{l}\text { Nofal et al. }{ }^{12} \\
2004\end{array}$} & 1 & 48; F & N/A & Positive & Low & High & $\begin{array}{l}\text { Zinc and amino } \\
\text { acids }\end{array}$ & $\begin{array}{l}\text { Four of the five had } \\
\text { DM, not specified } \\
\text { which. No other } \\
\text { diseases discussed. }\end{array}$ \\
\hline & 2 & $24 ; F$ & N/A & Positive & Normal & Normal & $\begin{array}{l}\text { Zinc and amino } \\
\text { acids }\end{array}$ & \\
\hline & 3 & 43; F & N/A & Positive & Normal & High & $\begin{array}{l}\text { Zinc and amino } \\
\text { acids }\end{array}$ & \\
\hline & 4 & 35; F & N/A & Positive & Low & Normal & $\begin{array}{l}\text { Zinc and amino } \\
\text { acids }\end{array}$ & \\
\hline & 5 & 51; F & $N / A$ & Positive & Normal & Normal & $\begin{array}{l}\text { Zinc and amino } \\
\text { acids }\end{array}$ & $\begin{array}{l}\text { HIV, hypothyroidism, } \\
\text { HTN }\end{array}$ \\
\hline $\begin{array}{l}\text { Abdallah et al., } \\
2005\end{array}$ & 1 & 46; F & Black & Positive & Normal & $N / A$ & Zinc & $\begin{array}{l}\text { SLE, prior HBV } \\
\text { infection, liver } \\
\text { cirrhosis, HTN, DM, } \\
\text { CAD }\end{array}$ \\
\hline $\begin{array}{l}\text { Najarian et al.,13 } \\
2006\end{array}$ & 1 & 48; F & N/A & Positive & Low & $N / A$ & Zinc & HIV-1 \\
\hline $\begin{array}{l}\text { Bentley et al., }{ }^{14} \\
2008\end{array}$ & 1 & $46 ; M$ & Black & Positive & Normal & Normal & Zinc & Haemophilia \\
\hline $\begin{array}{l}\text { Fielder et al., } \\
2008\end{array}$ & 1 & 42; F & N/A & Positive & $N / A$ & $N / A$ & Zinc & $\begin{array}{l}\text { HIV, hypothyroidism, } \\
\text { HTN }\end{array}$ \\
\hline Liu et al.,16 2008 & 1 & 56; F & White & Negative & Normal & Normal & N/A & $\begin{array}{l}\text { Crohn's disease, } \\
\text { psoriasis/ } \\
\text { psoriatic arthritis, } \\
\text { hypothyroidism, } \\
\text { fibromyalgia, HLD }\end{array}$ \\
\hline $\begin{array}{l}\text { Najarian et al., } \\
2008\end{array}$ & 1 & 65; F & Black & Positive & Low & N/A & Zinc & HTN \\
\hline $\begin{array}{l}\text { De Carvalho } \\
\text { Fantini et al..18 } \\
2008\end{array}$ & 1 & $24 ; F$ & White & Positive & Low & $N / A$ & Zinc & Liver cirrhosis \\
\hline
\end{tabular}


Table 1 continued.

\begin{tabular}{|c|c|c|c|c|c|c|c|c|}
\hline Study & Patient & $\begin{array}{l}\text { Age } \\
\text { (years); } \\
\text { Sex }\end{array}$ & Ethnicity & $\begin{array}{l}\text { HCV } \\
\text { Status }\end{array}$ & $\begin{array}{l}\text { Serum } \\
\text { zinc } \\
\text { level }\end{array}$ & $\begin{array}{l}\text { Serum } \\
\text { glucagon } \\
\text { level }\end{array}$ & Treatment & $\begin{array}{l}\text { Other diseases in } \\
\text { patient }\end{array}$ \\
\hline $\begin{array}{l}\text { Manzur et al., }{ }^{19} \\
2008\end{array}$ & 1 & 15; M & $\begin{array}{l}\text { Asian } \\
\text { (Pakistani) }\end{array}$ & Positive & N/A & N/A & $\begin{array}{l}\text { Topical } \\
\text { tacrolimus }\end{array}$ & N/A \\
\hline Wu et al., ${ }^{20} 2009$ & 1 & 32; F & $\begin{array}{l}\text { Asian } \\
\text { (Taiwanese) }\end{array}$ & Negative & Low & Normal & $\begin{array}{l}\text { Zinc and steroid } \\
\text { cessation }\end{array}$ & SLE, lupus nephritis \\
\hline \multirow[t]{2}{*}{ Nikam ${ }^{21} 2009$} & 1 & 38; M & N/A & Negative & Normal & N/A & Zinc & Down's Syndrome \\
\hline & 2 & 25; F & $\mathrm{N} / \mathrm{A}$ & Negative & N/A & N/A & Zinc & N/A \\
\hline $\begin{array}{l}\text { Zeller et al., } \\
2009\end{array}$ & 1 & $55 ; M$ & N/A & Positive & Low & N/A & Zinc & N/A \\
\hline $\begin{array}{l}\text { Halpern et al., }{ }^{23} \\
2009\end{array}$ & 1 & $50 ; M$ & N/A & Positive & High & Low & $\begin{array}{l}\text { IFN, ribavirin, } \\
\text { zinc }\end{array}$ & None \\
\hline \multirow{2}{*}{$\begin{array}{l}\text { Tabibian et al., }{ }^{24} \\
2010\end{array}$} & 1 & 19; F & Black & Positive & Low & $\mathrm{N} / \mathrm{A}$ & Zinc & None \\
\hline & 2 & 58; F & Black & Positive & Low & Normal & Zinc & $\begin{array}{l}\text { Anaemia, HTN, } \\
\text { fibromyalgia, s/p } \\
\text { cholecystectomy }\end{array}$ \\
\hline $\begin{array}{l}\text { Panda et al., }{ }^{25} \\
2010\end{array}$ & 1 & 68; M & N/A & Negative & Normal & N/A & $\begin{array}{l}\text { Zinc, topical } \\
\text { clobetasol } \\
\text { propionate }\end{array}$ & $\begin{array}{l}\text { DM, } \\
\text { hypertriglyceridaemia }\end{array}$ \\
\hline $\begin{array}{l}\text { Patel et al., }{ }^{26} \\
2010\end{array}$ & 1 & 53; F & N/A & Positive & Normal & N/A & Zinc & $\begin{array}{l}\text { Dermatomyositis, } \\
\text { iron-deficiency } \\
\text { anaemia }\end{array}$ \\
\hline $\begin{array}{l}\text { Kapoor et al., } \\
2011\end{array}$ & 1 & $44 ; M$ & N/A & Positive & Low & N/A & Zinc & $\begin{array}{l}\text { Lead toxicity, syphilis, } \\
\text { narcotic and alcohol } \\
\text { addiction }\end{array}$ \\
\hline $\begin{array}{l}\text { Ridder et al.., } \\
2011\end{array}$ & 1 & $17 ; \mathrm{F}$ & Black & Positive & Low & N/A & Zinc & N/A \\
\hline $\begin{array}{l}\text { Grauel et al.. }{ }^{29} \\
2012\end{array}$ & 1 & $59 ; M$ & Black & Positive & $\mathrm{N} / \mathrm{A}$ & N/A & Zinc & Liver cirrhosis \\
\hline \multirow[t]{5}{*}{$\begin{array}{l}\text { Raphael et al., } \\
2012 \\
\end{array}$} & 1 & 69; M & Black & Positive & N/A & N/A & $\begin{array}{l}\text { Clobetasol } \\
\text { and hand/foot } \\
\text { psoralen plus } \\
\text { UVA therapy }\end{array}$ & HIV, DM \\
\hline & 2 & 69; M & Black & Positive & N/A & N/A & $\begin{array}{l}\text { Topical } \\
\text { triamcinolone }\end{array}$ & None \\
\hline & 3 & $52 ; \mathrm{M}$ & Black & Positive & N/A & N/A & $\begin{array}{l}\text { Liver } \\
\text { transplantation }\end{array}$ & HIV \\
\hline & 4 & $40 ; M$ & Black & Positive & N/A & N/A & $\begin{array}{l}\text { Narrowband } \\
\text { UVB therapy }\end{array}$ & None \\
\hline & 5 & $55 ; M$ & Black & Positive & N/A & N/A & $\begin{array}{l}\text { Initially: } \\
\text { narrowband } \\
\text { UVB therapy. } \\
\text { After relapse and } \\
\text { hepatocellular } \\
\text { carcinoma } \\
\text { diagnosis: topical } \\
\text { triamcinolone, } \\
\text { IFN, and ribavirin }\end{array}$ & DM \\
\hline
\end{tabular}


Table 1 continued.

\begin{tabular}{|c|c|c|c|c|c|c|c|c|}
\hline Study & Patient & $\begin{array}{l}\text { Age } \\
\text { (years); } \\
\text { Sex }\end{array}$ & Ethnicity & $\begin{array}{l}\mathrm{HCV} \\
\text { Status }\end{array}$ & $\begin{array}{l}\text { Serum } \\
\text { zinc } \\
\text { level }\end{array}$ & $\begin{array}{l}\text { Serum } \\
\text { glucagon } \\
\text { level }\end{array}$ & Treatment & $\begin{array}{l}\text { Other diseases in } \\
\text { patient }\end{array}$ \\
\hline Yost et al.,31 2013 & 1 & $56 ; F$ & $N / A$ & Positive & Low & N/A & $N / A$ & Dermatomyositis \\
\hline $\begin{array}{l}\text { Pernet et al.,32 } \\
2014\end{array}$ & 1 & $50 ; M$ & $\begin{array}{l}\text { Black } \\
\text { (Cameroonian) }\end{array}$ & Negative & Normal & Normal & None & None \\
\hline $\begin{array}{l}\text { lyengar et al., } \\
2014\end{array}$ & 1 & 61; M & N/A & Positive & Low & N/A & $\begin{array}{l}\text { Zinc and HCV } \\
\text { treatment } \\
\text { recommended }\end{array}$ & $\begin{array}{l}\text { Intravenous drug } \\
\text { abuse }\end{array}$ \\
\hline $\begin{array}{l}\text { Jakubovic et al., }{ }^{9} \\
2015\end{array}$ & 1 & $34 ; F$ & N/A & Negative & Low & N/A & $\begin{array}{l}\text { Total parenteral } \\
\text { nutrition }\end{array}$ & $\begin{array}{l}\text { NAFLD, morbid } \\
\text { obesity, prior Roux- } \\
\text { en-Y gastric bypass }\end{array}$ \\
\hline Das et al., ${ }^{34} 2016$ & 1 & $30 ; \mathrm{F}$ & N/A & Negative & N/A & N/A & Zinc & None \\
\hline Hou et al., ${ }^{35} 2016$ & 1 & $60 ; M$ & N/A & Positive & Low & N/A & Zinc & $\begin{array}{l}\text { Psoriasis/psoriatic } \\
\text { arthritis, HTN, DM }\end{array}$ \\
\hline \multirow{2}{*}{$\begin{array}{l}\text { Pandit et al., } \\
2016\end{array}$} & 1 & $24 ; M$ & N/A & Negative & Low & $\mathrm{N} / \mathrm{A}$ & Zinc & None \\
\hline & 2 & 40; M & N/A & Negative & Low & N/A & Zinc & None \\
\hline $\begin{array}{l}\text { Botelho et al., }{ }^{36} \\
2016\end{array}$ & 1 & 31; F & $N / A$ & Positive & Low & N/A & Zinc & $\begin{array}{l}\text { Atopy, drug-induced } \\
\text { hepatitis from OCP }\end{array}$ \\
\hline $\begin{array}{l}\text { Srisuwanwattana } \\
\text { et al., }{ }^{37} 2017\end{array}$ & 1 & $64 ; F$ & $N / A$ & Negative & Normal & N/A & $\begin{array}{l}\text { Zinc, doxepin, } \\
\text { combined } 0.05 \% \\
\text { betamethasone } \\
\text { dipropionate/3\% } \\
\text { salicylic acid } \\
\text { ointment }\end{array}$ & None \\
\hline $\begin{array}{l}\text { Dabas et al.,38 } \\
2018\end{array}$ & 1 & $52 ; \mathrm{F}$ & $N / A$ & Positive & Low & N/A & $\begin{array}{l}\text { Zinc, sofosbuvir, } \\
\text { daclatasvir }\end{array}$ & N/A \\
\hline $\begin{array}{l}\text { Xiaoling et al., }{ }^{39} \\
2018\end{array}$ & 1 & 36; F & $\mathrm{N} / \mathrm{A}$ & Negative & Low & N/A & N/A & NAFLD, obesity, DM \\
\hline Xue et al., ${ }^{40} 2019$ & 1 & 35; M & $\begin{array}{l}\text { Asian } \\
\text { (Chinese) }\end{array}$ & Positive & N/A & N/A & $\begin{array}{l}\text { Zinc treatment } \\
\text { recommended }\end{array}$ & Chronic hepatitis B \\
\hline $\begin{array}{l}\text { Oikonomou et } \\
\text { al., }{ }^{41} 2019\end{array}$ & 1 & $66 ; F$ & $N / A$ & Positive & Low & N/A & $\begin{array}{l}\text { Prednisolone } \\
\text { and zinc }\end{array}$ & $\begin{array}{l}\text { HIV, liver cirrhosis, } \\
\text { COPD }\end{array}$ \\
\hline $\begin{array}{l}\text { Pathania et al., }{ }^{42} \\
2019\end{array}$ & 1 & 36; M & $N / A$ & Negative & N/A & N/A & $\begin{array}{l}\text { Zinc and topical } \\
\text { corticosteroid }\end{array}$ & $\begin{array}{l}\text { PE on rivaroxaban, } \\
\text { rivaroxaban-induced } \\
\text { NAE }\end{array}$ \\
\hline $\begin{array}{l}\text { Fukushima et } \\
\text { al.. }{ }^{43} 2020\end{array}$ & 1 & $55 ; \mathrm{F}$ & N/A & Negative & Low & N/A & Zinc & $\begin{array}{l}\text { Metastatic ovarian } \\
\text { cancer }\end{array}$ \\
\hline $\begin{array}{l}\text { Kumar et al., }{ }^{44} \\
2020\end{array}$ & 1 & 40; F & N/A & Negative & Normal & N/A & $\begin{array}{l}\text { B12 } \\
\text { supplementation } \\
\text { and } \\
\text { beclomethasone } \\
(0.1 \%)+\text { salicylic } \\
\text { acid (3\%) }\end{array}$ & $\begin{array}{l}\text { Vitamin B12 } \\
\text { deficiency }\end{array}$ \\
\hline
\end{tabular}


Table 1 continued.

\begin{tabular}{|c|c|c|c|c|c|c|c|c|}
\hline Study & Patient & $\begin{array}{l}\text { Age } \\
\text { (years); } \\
\text { Sex }\end{array}$ & Ethnicity & $\begin{array}{l}\mathrm{HCV} \\
\text { Status }\end{array}$ & $\begin{array}{l}\text { Serum } \\
\text { zinc } \\
\text { level }\end{array}$ & $\begin{array}{l}\text { Serum } \\
\text { glucagon } \\
\text { level }\end{array}$ & Treatment & $\begin{array}{l}\text { Other diseases in } \\
\text { patient }\end{array}$ \\
\hline \multirow[t]{5}{*}{$\begin{array}{l}\text { Parihar et al.. }{ }^{45} \\
2020\end{array}$} & 1 & $41 ; M$ & N/A & Negative & Low & N/A & $\begin{array}{l}\text { Zinc and } \\
\text { levothyroxine }\end{array}$ & $\begin{array}{l}\text { Hypothyroidism } \\
\text { following radioactive } \\
\text { iodine ablation for } \\
\text { Graves' disease }\end{array}$ \\
\hline & 2 & $27 ; F$ & N/A & Negative & Low & N/A & $\begin{array}{l}\text { Zinc and } \\
\text { levothyroxine }\end{array}$ & $\begin{array}{l}\text { Hypothyroidism } \\
\text { following radioactive } \\
\text { iodine ablation for } \\
\text { Graves' disease }\end{array}$ \\
\hline & 3 & 35; F & N/A & Negative & Low & N/A & $\begin{array}{l}\text { Zinc and } \\
\text { levothyroxine }\end{array}$ & $\begin{array}{l}\text { Hypothyroidism } \\
\text { following radioactive } \\
\text { iodine ablation for } \\
\text { Graves' disease }\end{array}$ \\
\hline & 4 & 48; F & N/A & Negative & Low & N/A & $\begin{array}{l}\text { Zinc and } \\
\text { levothyroxine }\end{array}$ & $\begin{array}{l}\text { Hypothyroidism } \\
\text { following total } \\
\text { thyroidectomy for } \\
\text { papillary carcinoma }\end{array}$ \\
\hline & 5 & 33; F & N/A & Negative & Low & N/A & $\begin{array}{l}\text { Zinc and } \\
\text { levothyroxine }\end{array}$ & $\begin{array}{l}\text { Primary } \\
\text { hypothyroidism }\end{array}$ \\
\hline $\begin{array}{l}\text { Shah et al., }{ }^{46} \\
2020\end{array}$ & 1 & 51; M & Black & Negative & Low & Normal & Zinc & $\begin{array}{l}\text { Sarcoidosis, } \\
\text { seronegative } \\
\text { chronic hepatitis } \\
\text { likely secondary to } \\
\text { sarcoidosis, DM }\end{array}$ \\
\hline $\begin{array}{l}\text { Davis et al., }{ }^{47} \\
2020\end{array}$ & 1 & 58; F & N/A & Positive & N/A & N/A & $\begin{array}{l}\text { Clobetasol, } \\
\text { zinc, and HCV } \\
\text { treatment } \\
\text { recommended }\end{array}$ & N/A \\
\hline $\begin{array}{l}\text { Koblinski* et al., } \\
2021\end{array}$ & 1 & 68; M & White & Positive & Low & High & $\begin{array}{l}\text { Clobetasol, } \\
\text { zinc, and } \mathrm{HCV} \\
\text { treatment } \\
\text { recommended }\end{array}$ & $\begin{array}{l}\text { Chronic venous } \\
\text { insufficiency, } \\
\text { HTN, tinea pedis, } \\
\text { intravenous drug } \\
\text { abuse }\end{array}$ \\
\hline
\end{tabular}

${ }^{*}$ Case from this paper.

CAD: coronary artery disease; COPD: chronic obstructive pulmonary disease; DM: diabetes mellitus; F: female; HBV: hepatitis B virus; HCV: hepatitis C virus; HLD: hyperlipidaemia; HTN: hypertension; IFN: interferon; M: male; N/A: not available; NAE: necrolytic acral erythema; NAFLD: non-alcoholic fatty liver disease; OCP: oral contraceptive pill; PE: pulmonary embolism; SLE: systemic lupus erythematosus; UVA: ultraviolet A; UVB: ultraviolet B.

The link between NAE and disruption in glucagon levels is often discussed, especially due to the similar entity NME. NME is the dermatosis associated with glucagonoma syndrome and presents as painful erythematous plaques that coalesce into bullous lesions; it affects the perioral and peri-genital regions as well as the extremities. ${ }^{5}$ While NAE and NME are similar in morphology, they have different body distributions. ${ }^{2}$ In addition, the two have similar findings under histopathological evaluation (as well as overlapping features with pellagra and acrodermatitis enteropathica), with neither having pathognomonic features. ${ }^{2,49,50}$ Both conditions can reveal parakeratosis, acanthosis, psoriasiform hyperplasia, and superficial epidermal necrosis depending on stage of disease and biopsy site. . $^{2,49}, 50$ These similarities 
demonstrate how clinical correlation as well as laboratory tests and imaging are necessary for a more definitive diagnosis. For further distinction between the two, patients with NME typically have glucagon levels $>500 \mathrm{pg} / \mathrm{mL},{ }^{5}$ while patients with NAE can have normal, ${ }^{10}$ low, ${ }^{16}$ or, less often reported, high levels of glucagon. ${ }^{12}$ The patient was found to have an elevated glucagon of 96 $\mathrm{pg} / \mathrm{mL}$ (normal: 8-57 pg/mL).

While this raised suspicion for NME, the patient's dermatologic findings were in a distribution consistent with NAE and he had a negative CT abdomen. Of the PubMed-indexed cases, only two cases reported by Nofal et al..$^{12}$ had high glucagon levels, so this is an infrequently reported (or potentially under-reported) finding. For those two cases, the actual values of their elevated glucagon were not listed, but both did have a negative CT scan of the pancreas as well as HCV. ${ }^{12}$ There is debate about whether NAE and NME are distinct entities or if NAE is truly a variant of NME, especially due to the similar histopathologic findings, ${ }^{12}$ and the mildly elevated glucagon level in the patient may suggest the latter. Whether the two pathologies are different or on a spectrum, glucagon levels should be checked when either is suspected. An elevated glucagon level warrants imaging to rule out glucagonoma. It has been hypothesised that in NAE, elevated serum glucagon induces inflammation through high levels of arachidonic acid and its metabolism. ${ }^{28}$
The patient's diagnosis of NAE had been missed for 12 years resulting in significant morbidity. It was affecting his quality of life and he had been exposed to unnecessary and costly treatment throughout this time. As the incidence and prevalence are unknown, it is unclear how many other patients with NAE may be misdiagnosed as well. It is important for providers to be able to recognise this entity, especially as it is treatable with zinc replacement and, if indicated, HCV therapy. ${ }^{2}$ This will help to avoid unnecessary morbidity for patients. Patients with $\mathrm{HCV}$ especially should be counselled about the cutaneous manifestations of HCV, including NAE, and told to report any concerns to their provider. As more cases are reported, the understanding of the epidemiology of NAE will increase and potentially lead to better recognition and care.

\section{CONCLUSION}

NAE is important to have on the differential diagnoses for patients with well-demarcated, erythematous acral plaques, especially in a patient with HCV. Misdiagnosis of NAE can lead to ineffective and unnecessary treatment and increased patient morbidity. If NAE is suspected, laboratory tests for HCV status as well as serum zinc and glucagon levels are warranted, and this case highlights elevated glucagon levels can be found in patients with NAE. Oral zinc is often a successful treatment and, if indicated, referrals to hepatology for HCV therapy are necessary.

\section{References}

1. el Darouti M, Abu el Ela M. Necrolytic acral erythema: a cutaneous marker of viral hepatitis C. Int J Dermatol. 1996;35(4):252-6.

2. Inamadar AC et al. Necrolytic acral erythema: current insights. Clin Cosmet Investig Dermatol. 2020;13:275-81.

3. Abdallah MA et al. Necrolytic acral erythema: a patient from the United States successfully treated with oral zinc. Arch Dermatol. 2005;141(1):85-7.

4. Bolognia J et al., Dermatology essentials, (2014) 2nd edition, Saint Louis, Missouri: Saunders.

5. Sandhu $S$ et al., Glucagonoma Syndrome, (2020) Treasure Island, Florida: StatPearls Publishing.

6. Pandit VS et al. Seronegative necrolytic acral erythema: a report of two cases and literature review. Indian Dermatol Online J. 2016;7(4):304-7.

7. Gomaa A et al. Hepatitis C infection in Egypt: prevalence, impact and management strategies. Hepat Med 2017;9:17-25.

8. Ilyas $\mathrm{S}$ et al. Necrolytic acral erythema: a rare entity. J Pak Assoc Dermatol. 2016;26(4):395-8.

9. Jakubovic BD et al. Zinc deficiency presenting with necrolytic acral erythema and coma. Am J Med. 2015;128(8):e3-4

10. Khanna VJ et al. Necrolytic acral erythema associated with hepatitis $\mathrm{C}$ : effective treatment with interferon alfa and zinc. Arch Dermatol. 2000;136(6):755-7.
11. Hivnor CM et al. Necrolytic acral erythema: response to combination therapy with interferon and ribavirin. J Am Acad Dermatol. 2004;50(5 Suppl):S121-4.

12. Nofal AA et al. Necrolytic acral erythema: a variant of necrolytic migratory erythema or a distinct entity? Int J Dermatol. 2005;44(11):916-21.

13. Najarian DJ et al. Zinc deficiency associated with necrolytic acral erythema. J Am Acad Dermatol. 2006;55(5 Suppl):S108-10.

14. Bentley D et al. Lack of classic histology should not prevent diagnosis of necrolytic acral erythema. J Am Acad Dermatol. 2009;60(3):504-7. 
15. Fielder LM et al. Necrolytic acral erythema: case report and review of the literature. Cutis. 2008;81(4):35560.

16. Liu A et al. Necrolytic acral erythema: a case not associated with hepatitis $\mathrm{C}$ infection. Dermatol Online J. 2008;14(6):10.

17. Najarian DJ et al. Hypozincemia and hyperzincuria associated with necrolytic acral erythema. Int Dermatol. 2008;47(7):709-11.

18. de Carvalho Fantini B et al. Necrolytic acral erythema successfully treated with oral zinc. Int J Dermatol. 2008;47(8):872-3.

19. Manzur A and Siddiqui AH. Necrolytic acral erythema: successful treatment with topical tacrolimus ointment. Int J Dermatol. 2008;47(10):1073-5.

20. Wu YH et al. Necrolytic acra erythema without hepatitis C infection. J Cutan Pathol. 2009;36(3):355-8.

21. Nikam BP. Necrolytic acral erythema seronegative for hepatitis $C$ virus-two cases from India treated with oral zinc. Int J Dermatol. 2009;48(10):1096-9

22. Zeller $\mathrm{S}$ et al. Necrolytic acral erythema associated with hepatitis $C$ and serum zinc deficiency. J Am Acad Dermatol. 2009;60(3):AB52.

23. Halpern AV et al. Necrolytic acral erythema: an expanding spectrum. Cutis. 2009;84(6):301-4.

24. Tabibian $\mathrm{J}$ et al. Necrolytic acral erythema as a cutaneous marker of hepatitis C: report of two cases and review. Dig Dis Sci. 2010;55:2735-43.

25. Panda S, Lahiri K. Seronegative necrolytic acral erythema: a distinct clinical subset? Indian J Dermatol. 2010;55(3):259-61.

26. Patel $U$ et al. Necrolytic acral erythema. Dermatol Online J. 2010;16(11):15.
27. Kapoor R, Johnson RA. Necrolytic acral erythema. N Engl J Med. 2011;364(15):1479-80.

28. Ridder $\mathrm{K}$ et al. Necrolytic acral erythema in an adolescent. Pediatr Dermatol. 2011;28(6):701-6.

29. Grauel E et al. Necrolytic acral erythema. J Drugs Dermatol. 2012;11(11):1370-1.

30. Raphael BA et al. Low prevalence of necrolytic acral erythema in patients with chronic hepatitis C virus infection. J Am Acad Dermatol. 2012;67(5):962-8.

31. Yost JM et al. Necrolytic acral erythema. Dermatol Online J. 2013;19(12):20709.

32. Pernet $C$ et al. Necrolytic acral erythema following hepatitis $B$ vaccination. $\mathrm{Br} J$ Dermatol. 2014;171(5):1255-6

33. Iyengar $\mathrm{S}$ et al. Necrolytic acra erythema masquerading as cellulitis. Dermatol Online J. 2014;20(11):15.

34. Das A et al. Necrolytic acral erythema in the absence of hepatitis $C$ virus infection. Indian J Dermatol. 2016:61(1):96-9.

35. Hou YC, Wu CY. Zinc-responsive necrolytic acral erythema in a patient with psoriasis: a rare case. Int J Low Extrem Wounds. 2016:15(3):260-2.

36. Botelho LF et al. Necrolytic acra erythema: a rare skin disease associated with hepatitis $C$ virus infection. An Bras Dermatol. 2016;91(5):649-51.

37. Srisuwanwattana P, Vachiramon V. Necrolytic acral erythema in seronegative hepatitis C. Case Rep Dermatol. 2017:9(1):69-73.

38. Dabas $\mathrm{G}$ et al. Necrolytic acral erythema leading to diagnosis of chronic hepatitis C. Dig Liver Dis. 2018;50(8):854.

39. Xiaoling $Y$ et al. Image gallery: seronegative necrolytic acral erythema. Br J Dermatol. 2018;179(2):e88.

40. Xue R et al. Necrolytic acral erythema in a Chinese patient with hepatitis $C$ and hepatitis B virus coinfection. An Bras Dermatol. 2019;94(4):446-8.

41. Oikonomou KG et al. Necrolytic acral erythema in a human immunodeficiency virus/hepatitis $C$ virus coinfected patient: a case report. World J Hepatol. 2019;11(2):226-33.

42. Pathania YS, Budania A. Rivaroxaban induced necrolytic acral erythema. Postgrad Med J. 2019;95(1128):563.

43. Fukushima $\mathrm{H}$ et al. Zinc-responsive necrolytic acral erythema in ovarian cancer. J Dermatol. 2020;47(7):e266-7.

44. Kumar $R$ et al. Necrolytic acral erythema in seronegative hepatitis $\mathrm{C}$ patient with vitamin B12 deficiency. Indian Dermatol Online J. 2020;11(2):278-9

45. Parihar AS et al. Necrolytic acral erythema in association with hypothyroidism. JAMA Dermatol. 2020;156(11):1268-70.

46. Shah $P$ et al. Necrolytic acral erythema in a patient with sarcoidosis. JAAD Case Rep. 2020;6(11):1162-4.

47. Davis S, Creditt A. Bilateral foot skin eruption in a hepatitis $C$ patient. Clin Pract Cases Emerg Med. 2020;4(3):491-2.

48. Sims OT et al. Racial disparities in hepatitis $C$ treatment eligibility. Ann Hepatol. 2017;16(4):530-7.

49. Plaza JA, Prieto VG, "Inflammatory skin conditions," Weidner $\mathrm{N}$ et al (eds.), Modern Surgical Pathology, (2009) 2nd edition, Philadelphia: Saunders.

50. Foss MG, Ferrer-Bruker SJ, Necrolytic migratory erythema, (2020) Treasure Island, Florida: StatPearls Publishing. 